\title{
Application Context
}

National Cancer Institute

\section{Source}

National Cancer Institute. Application Context. NCI Thesaurus. Code C41086.

A designation or description of the application environment or discipline in which a name

is applied or from which it originates. 